\title{
EVM FOR BLINDS: A NOVEL EMBEDDED DESIGN
}

\author{
Palak Gupta $^{1}$, Sona Sharma ${ }^{2}$, K.K. Singh ${ }^{3}$ \\ ${ }^{1}$ Electronic and Communication Engineering, Amity School of Engineering and Technology Lucknow, India \\ ${ }^{2}$ Electronic and Communication Engineering, Amity School of Engineering and Technology Lucknow, India \\ ${ }^{3}$ Electronic and Communication Engineering, Amity School of Engineering and Technology Lucknow, India
}

\begin{abstract}
Electronic Voting Machine for Blinds is a boon for the blind population for casting their vote without the presence of their helper/caretakers. It would provide the confidentiality of their vote. We have used the fingerprint identification method. Here, voters thumb impressions are used for identifying the voters. Once he has casted the vote and is authenticated the details of the person will be displayed on the LCD. If it does not match then the voter will not be permitted to vote. We have used symbols which has Braille format (a language for blinds). The blinds can figure out various parties represented by their symbols placed next to the buttons and can cast vote themselves by pressing the button with respect to the party of his/her choice. On pressing the button, the sound of buzzer can be heard which confirms the blind person that his vote has been casted. After all the votes have been casted, the authorized person will press the button and touch the RF card to RF module to access the total number of counts and the winner on LCD in a secured manner. This technique is beneficial as the same Braille symbolic representation can easily be read by normal population in the form of symbols.
\end{abstract}

Keywords—Finger Printing, RFID Module, Microcontroller, Sixth Sense Technology

\section{INTRODUCTION}

In a democratic country like India, a government is chosen by voting in an election, a way for an electorate to elect, i.e choose, among several candidates for rule. The objective of voting is to allow voters to exercise their right to express their choices regarding specific issues ${ }^{[2]}$.

The two types of system implemented for voting are:

1) Ballot Box

2) Electronic Voting Machine

A Ballot box is a square box shaped temporarily sealed container, with a narrow slot in the top sufficient to accept a ballot paper in an election.

Whereas an EVM consists of two parts: Balloting Unit and Controlling Unit. It reduces the time both in casting and declaring of result compared to ballot box.

The three types of problems faced in EVM system are:

1) Illegal Voting(Rigging):- One candidate can casts the votes of all the members or few amount of members in the electoral list illegally. This would result in the loss of votes for the other candidates participating and also increases the number votes to the candidate who performs this action. This can be done externally at the time of voting.

2) There is no provision of voting for the blind person, whose votes can play a significant role in formation of democratic government.

3) Security Problem:-

a) The program installed in the EVM can be changed and the results can be tampered after the voting. b) By replacing a small part of the machine with a look-alike component that can be silently instructed to steal a percentage of the votes in favor of a chosen candidate ${ }^{[8]}$. These instructions can be sent wirelessly from a mobile phone incorporating the applicable criteria that follow.

\section{MOTIVATION}

Considering all the disadvantages of existing EVM, we thought of enhancing the design of existing EVM which can overcome the problems during voting as:

1) Remedies to illegal voting: The issue of illegal voting can be eradicated by giving a unique id to each individual so that one person can cast his vote only once. This unique id can be "Fingerprint" of each individual ${ }^{[1]}$.

2) We can implement the symbols in Braille format (a language for blinds) across the buttons representing various parties. The blind population can sense these symbols and can cast their votes for the party of his/her choice without the presence of their helper/caretakers. It would provide the confidentiality of their vote.

3) Problem of security problem can be solved by:

a) Taking more care in keeping the EVMs safe and secure until the time of declaring the results, this can be done manually.

b) Immediately after voting, only the authorized person has the right to access the result by using RFID technology in a secured manner. 


\section{SOFTWARE USED}

\subsection{Proteus Software}

Proteus Pro 7.8 is a Virtual System Modelling that combines circuit simulation, animated components and microprocessor models to co-simulate the complete microcontroller based designs which are attached to the PC, switches and buttons. In summary, Proteus Pro 7.8 is the program to use to test the code written in c language using AVR Studio software.

\section{HARDWARE USED}

A list of Hardware used

\subsection{Microcontroller}

We have used ATmega8 microcontroller

1) FEATURES OF ATMEGA 8

a) Atmega 8 is a low-power AVR 8-bit microcontroller with high-performance

b) It can process up to 16 MIPS throughput at

c) It has $32 \times 8$ General Purpose

d) It has an internal calibrated RC oscillator

e) It has External and Internal Interrupt

f) It has data retention capability i.e. 20 years at $85 / 100$ years.

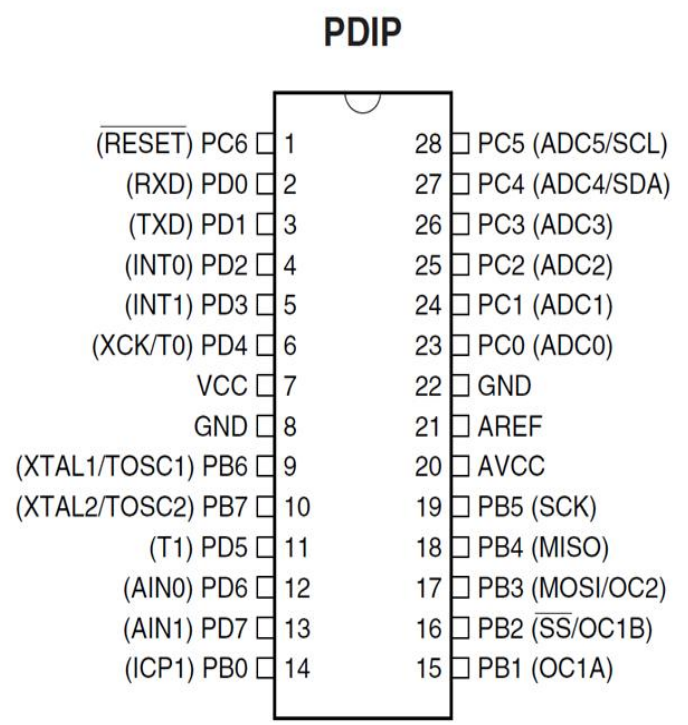

2) MEMORY SEGMENTSOF ATMEGA

a) Atmega8 has $8 \mathrm{~K}$ Bytes of In-System Selfprogrammable Flash program memory.

b) It has 512 Bytes of Electrically Erasable Programmable Read Only.

c) It has $1 \mathrm{~K}$ Byte of Internal Static Random Access Memory.

\subsection{Finger Print Module-R305}

We have used fingerprint scanner in this novel design. It is NITGEN Company based which is the leading manufacturer of FIM (Finger identification Module).
1) Features of finger print module-R305 are

a) It provides a combination of integrated image collection and algorithm chip together

b) It has low power consumption, low cost, small size, excellent performance

c) It uses professional optical technology, precise module manufacturing techniques

d) It gives good image processing capabilities and can capture up to resolution 500 dpi(dots per inch).

\subsection{Liquid Crystal Display(LCD)}

a) The LCD display is used to display the messages during the action ${ }^{[7]}$.

b) Here a 16x2 display is used, each character is made of $5 \times 7$ dot matrix.

c) Displays have built in backlight(blue or green diodes).

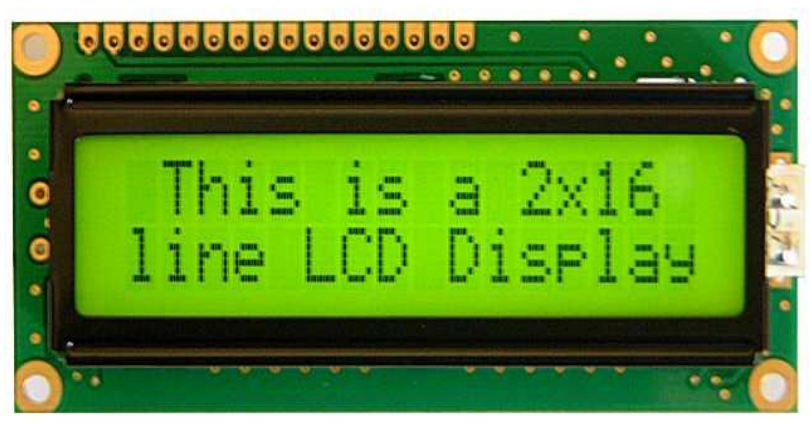

\subsection{Max 232}

a) MAX-Maxim company, 232- series number.

b) It is a 16-pin IC known as voltage converter or line convertor.

c) It can converts the RS-232 levels of the fingerprint scanner to the TTL voltage levels.

d) It provides the facility to connect two devices at a time to the MAX-232.

\subsection{RFID}

The Radio frequency identification is an automatic identification method which is used for storing and remotely retrieving data using devices called RFID tags. This technology requires cooperation of an RFID tag and an RFID reader

An RFID system consists of three components, namely ${ }^{[4]}$

a) An antenna or coil: The antenna emits radio wave signals to activate the tag and write and read data to it. They are the conduits between the transceiver and the tag which controls communication and the system's data acquisition. The field (electromagnetic ) produced by the antenna can be present constantly, if multiple tags are expected. Often the antenna is packaged with the decoder and transceiver to form a reader which can 
be configured as fixed-mount device. This reader emits radio waves depending upon its radio frequency used in ranges of anywhere from about one inch to 100 feet or more and power output. When an RFID passes near the electromagnetic zone, the antenna detects the readers activation signal. The reader then easily decodes the data encoded in the tag's integrated circuit and the data is send on to the host computer for furter processing

b) A transponder (RF tag): An RFID tag can be considered as an object that can be incorporated or applied into a person, product and animal, or for the purpose of tracking and identification using the radio waves.It consist of a microchip consisting of identifying information and an antenna which wirelessly transmits this data to a reader. The chip also contain a license plate number, serialized identifier, that uniquely identifies that item.

c) A transceiver(with decoder): The RF transceiver provides the RF energy used to power and activate the passive RFID tags. These transceiver may be enclosed in the cabinet of the reader or it may be considered as a separate piece of equipment. When transceiver is provided as a separate piece of equipment, then the transceiver is referred to as an RF module. The RF transceiver is used to modulates and controls the radio frequencies that the antenna receives and transmits.

\subsection{Buttons}

We have used E-switch type of switch.

\subsection{Buzzer}

\subsection{Power Supply}

This novel design uses a regulated $5 \mathrm{~V}, 500 \mathrm{~mA}$ power supply.7805 three terminal voltage regulator is used for voltage regulation.

\section{WORKING OF EVM}

Our EVM can be divided into three sections:

1) First section is dealing with the fingerprinting technology for authentication.

2) Second section is dealing with voting machine which is designed in a way so that both the blind and normal population can cast their votes.

3) Third section consists of secure access of result using RFID technology.

In this novel design, we have used the fingerprint identification method. Here, voters thumb impressions are used for identifying the validity of voters. During voting when the voter keeps his/her thumb in the scanner, the system will check whether it matches with pre stored impressions in the database ${ }^{[3]}$. If it matches then a buzzer will be heard to the blind person which indicates him that he is authenticated to cast his vote and the details of the person will be displayed on the LCD. If it does not match then the voter will not able to cast vote. We have used symbols which has Braille format (a language for blinds) ${ }^{[6]}$. The blinds can figure out various parties represented by their symbols placed next to the buttons and can cast vote themselves by pressing the button with respect to the party of his/her choice. On pressing the button, the sound of buzzer can be heard which confirms the blind person that his vote has been casted.

After all the votes have been casted, the authorized person will press the button and touch the RF card to RF module to access the total number of counts and the winner on LCD is displayed in a secured manner.

\section{FUTURE SCOPE}

In today's scenario, Sixth Sense Technology is considered as one of the leading technology, so we thought of using this technology in our concept i.e. in EVM for blinds. As 'Sixth Sense Technology' is used to build bridge between the digital world and the physical world it can be used for the same. We would like to work on this novel design and will make India a digital country.

\section{ADVANTAGES}

1) It is cost effective

2) It is low power consumption

3) It is economical

4) It requires less manpower

5) It is time conscious, as less time is consumed in voting and counting

6) It avoids illegal voting.

7) It saves cost of tranportation due to its compact size.

\section{CONCLUSION}

This novel design can be used for voting since it overcomes all the drawbacks of ordinary voting machine.

1) Its main advantage is that since fingerprints of every person is unique and hence this system completely reduces the chance of invalid votes and provides a higher level of security to an identification process. It also eliminates the burden and bulk of carrying id cards or remembering pins.

2) This this novel design is beneficial as the same Braille symbolic representation can easily be read by normal population in the form of symbols.

3) It provide additional security as the result of voting cannot be modified by illegal means as the system is secured by RFID. Only the authorized person has the right to access the result of voting.

4) This system can be manufactured simply as well as it is cheap and at the same time it isproviding a combination of authentication of valid user, a boon for blind population and secured access to result of voting.

5) The novel design "ELECTRONIC VOTING MACHINE for BLINDS: A NOVEL EMBEDDED $D E S I G N$ " has been successfully designed and tested. 
6) This can bring a revolutionary change in the election procedures.

7) As the normal EVM is the burning issue in recent days, this can be a solution for all the problems.

8) Not only in INDIA but also in other countries the face of the election procedure can be changed drastically using this technology.

\section{REFERENCES}

[1] Development Of Ant rigging Voting System Using Fingerprint Description.

[2] Engr Rana Muhammad Shakee,"Biometric Voting Machine".

[3] Engr Rana Muhammad Shakee,"Fingerprint based Electronic Voting Machine".

[4] Jhani Bhasha Shaik1, Mazhar Hussain Shaik,"Voter Identification and Detection on System using RFID and GSM to stop rigging in the elections", INTERNATIONAL JOURNAL OF INNOVATIVE RESEARCH IN ELECTRICAL, ELECTRONICS, INSTRUMENTATION AND CONTROL ENGINEERING Vol. 2, Issue 6.

[5] B.FarhathAnjum, M.Deepa, Mrs.C.N. Kalaivani, "Advanced Microcontroller Based Bio-Metric Authentication Voting Machine", IOSR Journal of Engineering (IOSRJEN) ISSN (e): 2250-3021, ISSN (p): 2278-8719 Vol. 04, Issue 05 (May. 2014), \|V1\| PP 29-40.

[6] Introduction of Braille Signage Feature in Electronic Voting Machine.

[7] Rohit Kumar and Rajdeep Chauhan,"Electronic Voting Machine: Electronic Club IIT Kanpur"

[8] Hari K. Prasad* J. Alex Halderman† Rop Gonggrijp Scott Wolchok† Eric Wustrow† Arun Kankipati* Sai Krishna Sakhamuri* Vasavya Yagati*,"Security Analysis of India's Electronic Voting Machine"

[9] Muhammad Ali Mazidi, Sarmad Naimi, Sepehr Naimi,"The AVR Microcontroller and Embedded System".

[10] Scott Wolchok, Eric Wustrow, J. Alex Halderman, Hari K. Prasad, Arun Kankipat,i Sai Krishna Sakhamuri Vasavya Yagati, Rop Gonggrijp," Security Analysis of India's Electronic Voting Machines". 